\title{
Upgraded photon calorimeter with integrating readout for the Hall A Compton polarimeter at Jefferson Lab
}

\author{
M. Friend for the HAPPEX Collaboration \\ Carnegie Mellon University, Department of Physics - 5000 Forbes Ave \\ Pittsburgh, PA 15213, USA
}

ricevuto il 12 Ottobre 2011; approvato il 5 Maggio 2012 pubblicato online il 26 Giugno 2012

\begin{abstract}
Summary. - The photon arm of the Compton polarimeter in Hall A of Jefferson Lab has been upgraded to allow for electron beam polarization measurements with better than $1 \%$ accuracy. The data acquisition system (DAQ) now includes an integrating mode, which eliminates several systematic uncertainties inherent in the original counting-mode DAQ. The photon calorimeter has been replaced with a Cedoped $\mathrm{Gd}_{2} \mathrm{SiO}_{5}$ crystal, the bright output and fast response of which are ideal for measurements using the new integrating method. The upgraded system was used to continuously measure the electron beam polarization during HAPPEX-III, and the results of this high precision measurement are presented.
\end{abstract}

PACS 29.90+r - Other topics in elementary-particle and nuclear physics experimental methods and instrumentation.

\section{1. - Introduction}

New electron scattering parity violation experiments require a better than $1 \%$ absolute measurement of the beam polarization. This includes HAPPEX-III [1], which ran in Hall A of Jefferson Lab [2,3] in 2009. The Hall A Compton polarimeter was upgraded [4] as preparation for HAPPEX-III and other Hall A experiments, in order to reach these stringent polarimetry requirements.

In Compton polarimetry, longitudinally polarized electrons scatter from circularly polarized laser light, and there is a different scattering cross-section depending on the relative polarizations of the electrons and photons. The measured experimental asymmetry (for a single laser polarization) is

$$
A_{\exp }=\left(S^{+}-S^{-}\right) /\left(S^{+}+S^{-}\right),
$$

where $S^{+(-)}$is the (integrated or counted) signal for a positive- (negative-) electron helicity state, and the helicity is flipped at $30 \mathrm{~Hz}$. This measured value can be used to 
determine the electron beam polarization, since

$$
A_{\mathrm{exp}}=A_{\mathrm{th}} P_{\mathrm{e}} P_{\gamma} \quad \Rightarrow \quad P_{\mathrm{e}}=A_{\mathrm{exp}} /\left(P_{\gamma} A_{\mathrm{th}}\right)
$$

where $A_{\text {th }}$ is the calculated theoretical asymmetry for Compton scattering at the given kinematics, $P_{\mathrm{e}}$ is the electron beam polarization, and $P_{\gamma}$ is the photon polarization.

In the Hall A Compton polarimeter, circularly polarized photons are locked in resonance in a high-finesse Fabry-Pérot cavity in the center of a chicane. The electron beam is sent into the chicane by dipole magnets, allowing electrons to scatter with photons in resonance in the cavity. Scattered photons are detected in the photon calorimeter, a scintillating crystal read out by a photo-multiplier tube (PMT). Since only 1 electron in $10^{9}$ scatters, and the rest are bent back to the beamline by the final two dipoles, the Compton polarimeter is non-invasive and can run continuously during experiments.

\section{2. - Hall A Compton polarimeter photon arm upgrade}

An energy-weighted measurement yields a higher measured asymmetry, since the Compton scattered photon analyzing power spectrum has the form shown in fig. $1 \mathrm{~b}$. Integration of the detector signal yields an energy-weighted measurement, since, on average, higher energy photons deposit more energy in the photon calorimeter than lower energy ones.

The original Hall A Compton polarimeter used a counting-mode data acquisition system (DAQ), which counts all photon triggers beyond some set discriminator threshold [5]. This contrasts with the upgraded photon DAQ [4], which integrates all PMT output within every $33 \mathrm{~ms}$ single-electron-helicity window. Integration has several benefits, including the elimination of threshold sensitivity, dead-time, and pileup, all of which contribute significant systematic uncertainties to a stand-alone photon-arm counting-mode measurement. Integration does increase sensitivity to non-linearities and gain shifts, as well as ADC pedestal noise, which are effects which must be taken into account during analysis.

$2 * 1$. Upgraded photon detector. - The upgraded photon calorimeter consists of a $15 \mathrm{~cm}$ long, $6 \mathrm{~cm}$ diameter Ce-doped $\mathrm{Gd}_{2} \mathrm{SiO}_{5}$ (GSO) crystal read out by a PMT and base which have been tested and designed for linear response using a specially built light-emitting diode pulser [6]. The scintillating crystal produces $\sim 450$ optical photons per $\mathrm{MeV}$ (electron equivalent), and pulses which are $\sim 80 \mathrm{~ns}$ full width at half maximum.

2:2. Upgraded photon DAQ. - The upgraded integrating DAQ uses a customized SIS3320 FADC which runs in two simultaneous modes: an accumulator (integrating) mode and a triggered mode. The integrating mode allows for a stand-alone photon measurement, while the triggered mode is used to monitor systematic effects in the scattered photon spectrum.

\section{3. - HAPPEX-III beam polarization results}

To measure $A_{\exp }$ from eq. (1), the detected photon signal is integrated over each electron-helicity window. A local background (measured when the cavity is unlocked) is also subtracted for each cavity-locked period. There was a $1 \%$ gain shift between cavitylocked and -unlocked during HAPPEX-III, which was taken into account during analysis and contributed a $0.37 \%$ systematic uncertainty to the polarization measurement. 


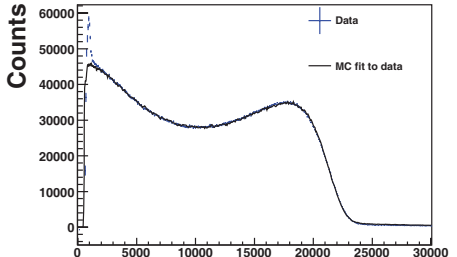

ADC Response (raus)

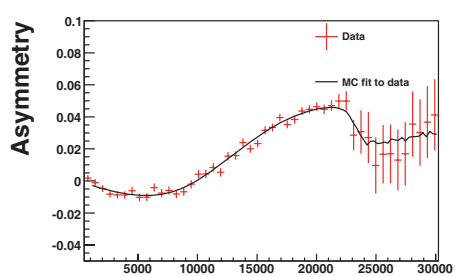

ADC Response (raus)

Fig. 1. - A measured Compton photon energy spectrum (a) and measured Compton asymmetry as a function of ADC response (b).

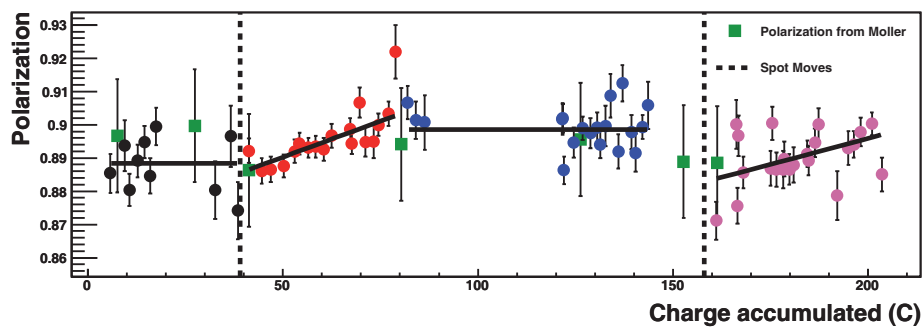

Fig. 2. - Measured polarization vs. HAPPEX-III charge accumulated: dots are from the Compton polarimeter integrating DAQ (error bars are statistical only), vertical dashed lines mark when the laser spot was moved at the accelerator source, solid lines are linear fits to the Compton data, and squares are from the Hall A Møller polarimeter [2] (Møller error bars include a $1.7 \%$ systematic error).

The photon polarization, $P_{\gamma}$ from eq. (2), was $99.02 \pm 0.80 \%$ and was stable throughout HAPPEX-III. The analyzing power, $A_{\text {th }}$ from eq. (2), was calculated using the results of a GEANT4 simulation. A fit of the simulation to the triggered data with only two free parameters is shown in fig. 1a. An overlay of the measured Compton asymmetry as a function of deposited energy and the GEANT4 simulated Compton asymmetry (without free parameters) is given in fig. 1b. An energy-weighted asymmetry calculated by summing over the simulated deposited-photon energies was used for $A_{\mathrm{th}}$, where the analyzing power contributed a $0.33 \%$ systematic uncertainty to the polarization measurement.

During HAPPEX-III, the beam polarization was measured to be $[89.41 \pm 0.05$ (stat) \pm 0.84 (syst) \pm 0.18 (gaps) $] \%$ using the upgraded Compton polarimeter, and the beam polarization is plotted as a function of HAPPEX-III charge accumulated in fig. 2.

\section{REFERENCES}

[1] Ahmed Z. et al. (HAPPeX Collaboration), Phys. Rev. Lett., 108 (2011) 102001.

[2] Alcorn J. et al., Nucl. Instrum. Methods A, 522 (2004) 294.

[3] Leemann C. W., Douglas D. R. and Krafft G. A., Annu. Rev. Nucl. Part. Sci., 51 (2001) 413.

[4] Friend M. et al., Nucl. Instrum. Methods A, 676 (2012) 96.

[5] Escoffier S. et al., Nucl. Instrum. Methods A, 551 (2005) 563.

[6] Friend M., Franklin G. and Quinn B., Nucl. Instrum. Methods A, 676 (2011) 66. 